\title{
The Form Factors of the Nucleons
}

\author{
C.F. Perdrisat \\ the College of William and Mary, Williamsburg, VA 23187
}

There has been much activity in the measurement of the elastic electromagnetic proton and neutron form factors in the last decade, and the quality of the data has been greatly improved by performing double-polarization experiments, in comparison with with previous unpolarized cross section data. Here we will review the experimental data base in view of the new results for the proton and the neutron, obtained at MIT-Bates, JLab and MAMI. The rapid evolution of phenomenological models triggered by these highprecision experiments will be discussed. In particular, the possibility that the proton is non-spherical in its ground state, and that the transverse charge density are model independently defined in the infinite momentum frame. Likewise, flavor decomposition of the nucleon form factors into dressed $u$ and $d$ quark form factors, may give information about the quark-diquark structure of the nucleon. The current proton radius "crisis" will also be discussed.

\section{Elastic ep cross section and form factors}

For almost fifty years following the ground braking work of R. Hofstadter [1], elastic $e p$ cross section measurements have been the only method available to obtain the Born approximation, single photon exchange, invariant form factor $F_{1}$ and $F_{2}$, or the related Sachs form factors, $G_{E}$ and $G_{M}$. As was shown by Foldy [2], the most general form for the hadronic current for a spin $\frac{1}{2}$-nucleon, $\mathcal{J}_{\text {hadronic }}$, satisfying relativistic invariance and current conservation with internal structure is:

$$
\mathcal{J}_{\text {hadronic }}^{\mu}=i e \bar{\nu}\left(p^{\prime}\right)\left[\gamma^{\mu} F_{1}\left(Q^{2}\right)+\frac{i \sigma^{\mu \nu} q_{\nu}}{2 M_{p}} F_{2}\left(Q^{2}\right)\right] \nu(p), \text { where }
$$

where $Q^{2}=\vec{q}^{2}-\omega^{2}=-q_{\mu}^{2}$, is the negative of the square of the invariant mass $q_{\mu}^{2}$ of the virtual photon exchanged in the one-photon description of elastic ep scattering.

The Lab cross section is then:

$$
\frac{d \sigma}{d \Omega_{e}}=\frac{\overline{\mathcal{M}}^{2}}{64 \pi^{2}}\left(\frac{E_{2}}{E_{1}}\right)^{2} \frac{1}{M_{p}} \text { with } \overline{\mid \mathcal{M}}^{2}=\frac{1}{Q^{2}} \overline{\mid \ell \cdot \mathcal{J}}^{2}
$$

where $\ell$ is the electromagnetic current. The lab cross section is then:

$$
\frac{d \sigma}{d \Omega_{e}}=\left(\frac{d \sigma}{d \Omega}\right)_{M o t t} \times\left\{F_{1}^{2}\left(Q^{2}\right)+\tau\left[F_{2}^{2}\left(Q^{2}\right)+2\left(F_{1}\left(Q^{2}\right)+F_{2}\left(Q^{2}\right)\right)^{2} \tan ^{2} \frac{\theta_{e}}{2}\right]\right\}
$$

where $\left(\frac{d \sigma}{d \Omega}\right)_{M o t t}$ is the Mott cross section given by:

$$
\left(\frac{d \sigma}{d \Omega}\right)_{M o t t}=\frac{\alpha^{2}}{4 E_{\text {beam }}^{2} \sin ^{4} \frac{\theta}{2}} \frac{E_{e}}{E_{\text {beam }}} \cos ^{2} \frac{\theta}{2}
$$


with the recoil factor $\frac{E_{e}}{E_{\text {beam }}}=\left(1+\frac{2 E_{\text {beam }}}{m} \sin ^{2} \frac{\theta_{e}}{2}\right)^{-1}$

The cross section can be written in a much simpler form, without interference term, with the Sachs form factors $G_{E}$ and $G_{M}$ defined as

$$
\begin{gathered}
G_{E}=F_{1}-\tau F_{2} \\
G_{M}=F_{1}+F_{2}: \\
\frac{d \sigma}{d \Omega}=\frac{d \sigma}{d \Omega}{ }_{M o t t} \frac{G_{E}^{2}+\frac{\tau}{\varepsilon} G_{M}^{2}}{1+\tau},
\end{gathered}
$$

$\tau=\frac{Q^{2}}{4 m_{p}^{2}}$ and $\varepsilon=\left[1+2(1+\tau) \tan ^{2} \frac{\theta_{e}}{2}\right]^{-1}$, where $\theta_{e}$ is the lab electron scattering angle, leading to a simple separation method for $G_{E_{p}}^{2}$ and $G_{M_{p}}^{2}$, referred to as Rosenbluth (or LT) separation. Note that this technique give the squares of the form factors and is therefore insensitive to $G_{M_{p}}$ near $Q^{2}=0$, and insensitive to $G_{E_{p}}$ for large $Q^{2}$.

All elastic ep data for $G_{E_{p}}$ and $G_{M_{p}}$ obtained by Rosenbluth separation for $Q^{2}>0.05$ $\mathrm{GeV}^{2}$ are shown separately in Figs. 1 and 2; the form factors have been divided by the dipole form factor, $G_{D}=\frac{1}{\left(1+\frac{Q^{2}}{0.71}\right)^{2}}, Q^{2}$ in $\mathrm{GeV}^{2}$.

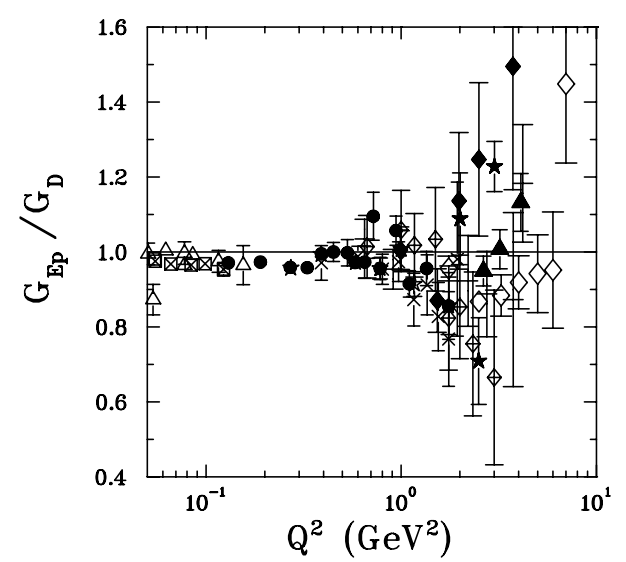

Figure 1: Electric form factor of the proton from cross section measurements, and Rosenbluth separation. Notice significant increase of the error bars above $1 \mathrm{GeV}^{2}$.

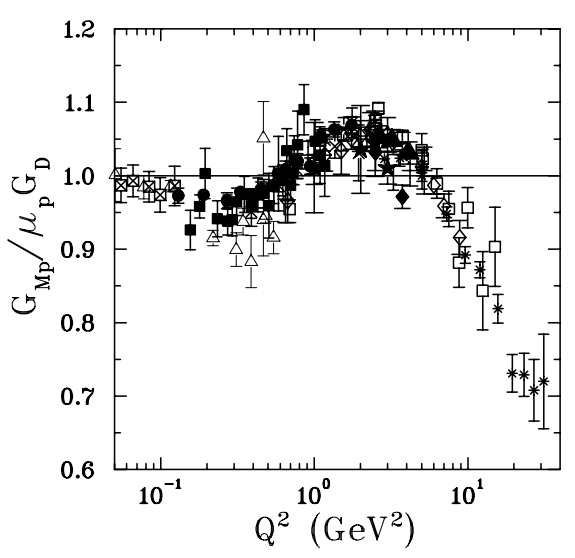

Figure 2: Magnetic form factor of the proton from cross section measurements and Rosenbluth separation. Notice consistency of the data up to at least $10 \mathrm{GeV}^{2}$.

References for the data in Figs. 1 and 2 are in $[5,6]$.

\section{Recoil Polarization Method}

The relationship between the Sachs electromagnetic form factors and the degree of polarization transfer in ${ }^{1} H\left(\vec{e}, e^{\prime} \vec{p}\right)$ scattering was first developed by Akhiezer and Rekalo [3], and later discussed in more detail by Arnold, Carlson, and Gross [4].

For single photon exchange, the transferred polarization can be written in terms of the Sachs form factors as: 


$$
\begin{aligned}
P_{n} & =0 \\
h P_{e} P_{l} & =h P_{e}\left(\frac{E_{e}+E_{e}^{\prime}}{M}\right) \frac{\sqrt{\tau(1+\tau)} G_{M p}^{2}\left(Q^{2}\right) \tan ^{2} \frac{\theta_{e}}{2}}{G_{E p}^{2}\left(Q^{2}\right)+\frac{\tau}{\epsilon} G_{M p}^{2}\left(Q^{2}\right)} \\
h P_{e} P_{t} & =h P_{e} \frac{2 \sqrt{\tau(1+\tau)} G_{E p} G_{M p} \tan \frac{\theta_{e}}{2}}{G_{E p}^{2}\left(Q^{2}\right)+\frac{\tau}{\epsilon} G_{M p}^{2}\left(Q^{2}\right)}
\end{aligned}
$$

for the normal, in-plane longitudinal and transverse polarization components $P_{n}, P_{\ell}$ and $P_{t}$, respectively; $h= \pm$ stands for the two possible orientations of the electron beam helicity, and $P_{e}$ for the electron beam polarization.

For each $Q^{2}$, a single measurement of the azimuthal angular distribution of the proton scattered in a secondary target (described later) gives both the longitudinal and transverse polarizations. Combining Eqs. 8 and 9 give:

$$
\frac{G_{E p}}{G_{M p}}=-\frac{P_{t}}{P_{l}} \frac{\left(E_{e}+E_{e}^{\prime}\right)}{2 M} \tan \frac{\theta_{e}}{2}
$$

thus the ratio of electric to magnetic form factors of the proton is obtained directly from a simultaneous measurement of the two recoil polarization components. The kinematic factors in Eq. 10 are typically known to a precision far greater than the statistical precision of the recoil polarization components.

The striking disagreement of the polarization data with the Rosenbluth results is illustrated in Fig. 3, to be compared with Figs. 1 and 2.

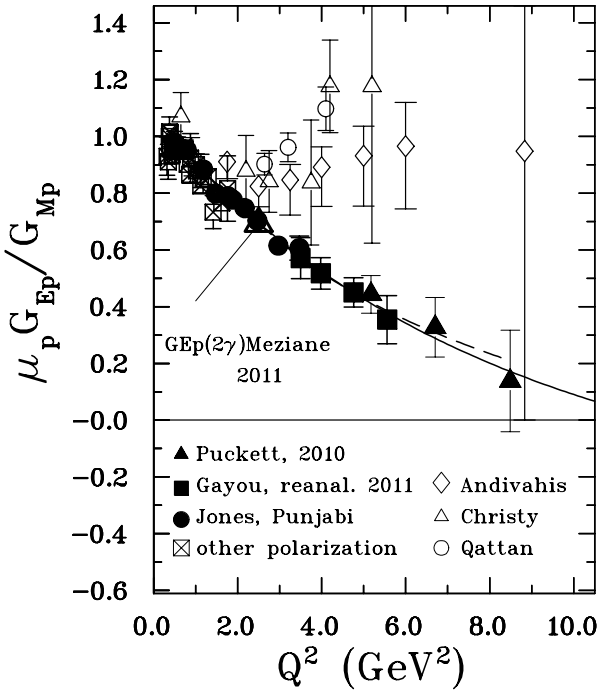

Figure 3: The ratio $\mu_{p} G_{E_{p}} / G_{M_{p}}$ obtained in polarization transfer experiments shown as filled symbols, refs. [7, 8, 9, 10, 11, 12]. Also shown as empty symbols are the recent Rosenbluth results of refs. $[13,14,15]$.

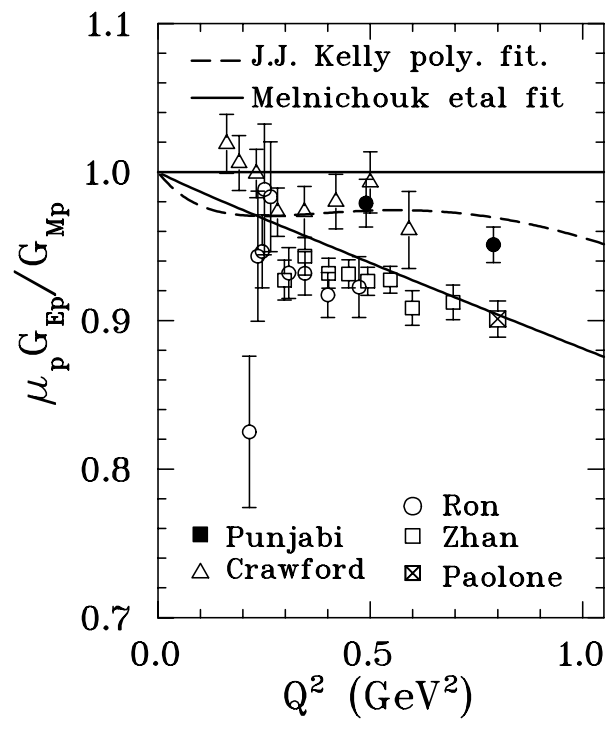

Figure 4: The same ratio for $\mathrm{Q}^{2}$ smaller than $1 \mathrm{GeV}^{2}$, all obtained in double polarization experiments, ref. $[9,16,17,18,19]$

Another disagreement, this time about the proton size or rms radius, was recently revealed by comparing the results of a muonic hydrogen Lamb shift experiment at PSI 
[20], with previous hydrogen Lamb shift, as well as elastic ep form factor measurements. These experiments indicate a $5 \sigma$ discrepancy between the rms radii from the muonic hydrogen, and the average of all previous measurements. These results have led to several proposals for experiments at PSI (elastic muon scattering, $0.004<Q^{2}<0.08 \mathrm{GeV}^{2}$ ), and elastic electron scattering at JLab Hall A (data taken, $0.25<Q^{2}<0.5 \mathrm{GeV}^{2}$ ) and B (approved in 2012, $0.0001<Q^{2}<0.02 \mathrm{GeV}^{2}$ ).

\section{Discussion}

\subsection{Two-photon exchange}

Soon after the publication of the results of the second JLab $G_{E_{p}} / G_{M_{p}}$, a first paper [21] suggested that the irreconcilable difference between the results of Rosenbluth and double polarization experiments was due to the two-photon contribution; this term has traditionally been included in radiative correction calculation in the infrared limit only. The effect of the exchange of two photons of similar momenta is to correct the Rosenbluth ratio downwards. After this paper many others have been published (for example [22, 23, $25]$ ), the reason for this plurality being the difficulty in evaluating such diagrams, as the proton in the intermediate state is off-shell, and can be exited to a number of isobaric states. Recently, at Jlab, a measurement of the $G_{E_{p}} / G_{M_{p}}$ ratio was done, at a constant $\mathrm{Q}^{2}$ of $2.5 \mathrm{GeV}^{2}$ but for 3 values of $\epsilon$ [11], to verify the prediction that only the Rosenbluth results would be significantly affected by two-photon exchange, not double polarization results, which are ratios of ratios (see Eqs. 8, 9 and 10) leading to near cancellation of the effect.

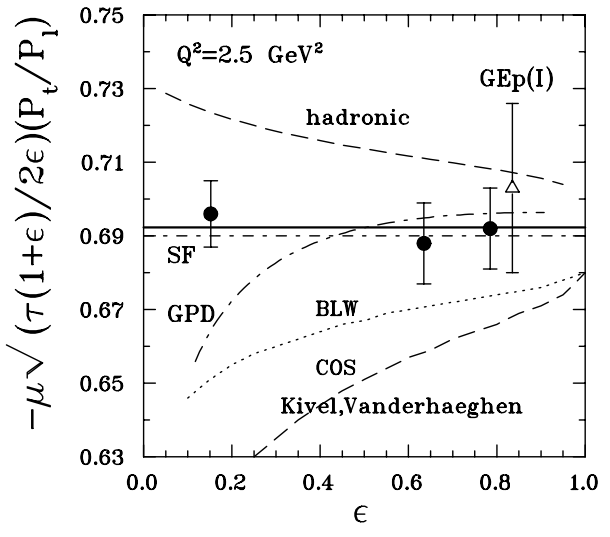

Figure 5: Plotted in ordinate is the quantity $-\mu_{p} \sqrt{(\tau(1+\epsilon) / 2 \epsilon)}$ which, in Born approximation, is equal to $G_{E_{p}} / G_{E_{M}}$; error bars include systematics uncertainties. Curves: "hadronic" [22], "GPD" [23], "COZ BLW" [24] and "SF" [25].

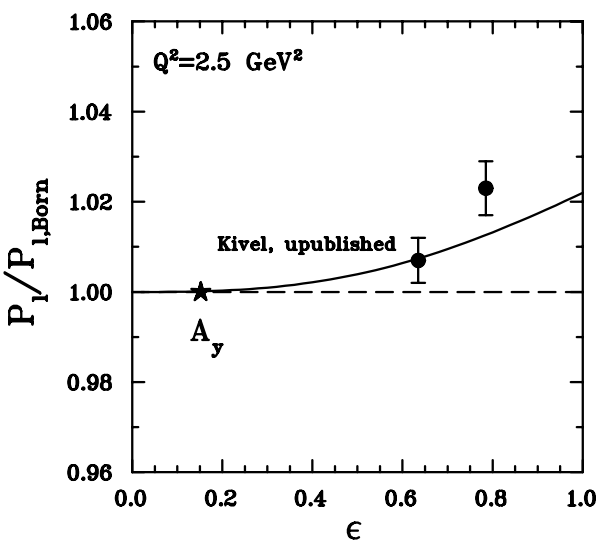

Figure 6: Longitudinal proton polarization, $P_{\ell}$, divided by the Born value $P_{\ell_{\text {Born }}}$ calculated from $G_{E_{p}} / G_{E_{M}}$ in Fig. 5. The required analysis power $A_{y}$ was obtained from the lowest $\epsilon$ point. Curve: [26]

The results for are shown in Fig. 5, [11]; the ratio is constant at the \pm 0.01 level, confirming predictions. Also shown in Fig. 6 is the ratio of $P_{\ell}$ to $P_{\ell} / P_{\ell_{B o r n}}$, which requires knowledge of the absolute value of the analyzing power; that was obtained from one of the 
data point, and the assumption that at constant $\mathrm{Q}^{2}$ (therefore constant p-momentum) the analyzing power is the same for all 3 points when appropriate cuts are made on the phase space of the proton so that the angle-momentum distribution is the same for all points. The two points at larger $\varepsilon$ differ from 1 by to $3 \sigma$ 's. Note that for the ratio shown in Fig. 5 the analyzing power cancels out.

\subsection{Flavor separation}

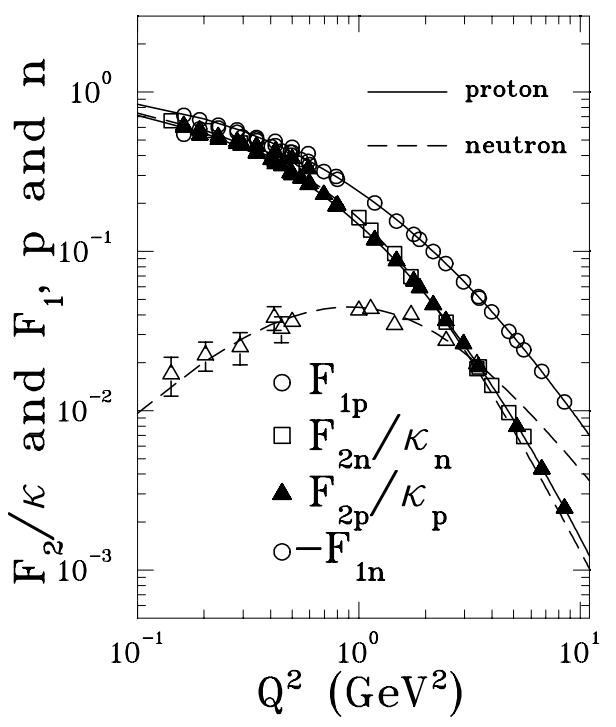

Figure 7: The $F_{1}$ and $F_{2}$ form factors calculated from the measured $G_{E}$ and $G_{M}$ values for proton and neutron. The curves are fits (polynomial over polynomial [28]), except for $F_{2_{n}}$, which is a simple polynomial. For $G_{E_{n}}$ no data exist above $3.5 \mathrm{GeV}^{2}$.

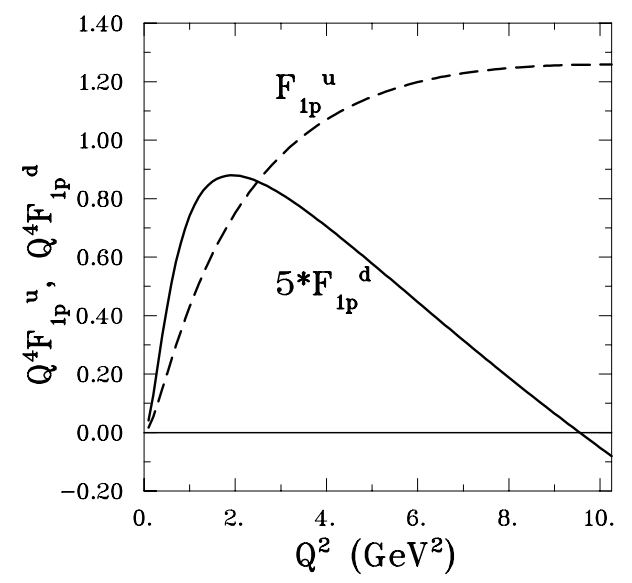

Figure 8: The flavor separated form factors of $F_{1}^{u}$ and $F_{1}^{d}$ obtained from the fits in Fig. 7 .

With the assumption of isospin symmetry for the $F_{1}^{u, d} F_{2}^{u, d}$ form factors of the dressed quarks in the proton, $F_{1 n}^{d}=F_{1 p}^{u}, F_{1 n}^{u}=F_{1 p}^{d}$, and similar relations for $F_{2}$, the dressed 
quark form factors can be obtained from $F_{1 p}^{u}=2 F_{1 p}+F_{1 n}$ and $F_{1 p}^{d}=F_{1 p}+2 F_{1 n}$, and similar relations for $F_{2}$. Results from such a separation have been recently published [27], obtaining the required values of the four form factors, $F_{1 p}, F_{2 p}, F_{1 n}$ and $F_{2 n}$ at the same $\mathrm{Q}^{2}$ by interpolation of the Sachs form factors. In Figs. 7 the results of fitting the nucleon $F_{1_{p, n}}$ and $F_{2_{p, n}}$ form factors with polynomials ratios as suggested by Kelly, and also extrapolating beyond the $\mathrm{Q}^{2}$ for $G_{E n}$; this procedure is justified by the remarkable smoothness of the fits to $F_{1_{p, n}}$ and $F_{2_{p, n}}$ (see Fig. 7). The separated dressed quark form factors, obtained as described above, and multiplied by $\mathrm{Q}^{4}$, are shown in Figs. 8 and 9. One interesting feature of Fig. 8 is the zero crossing for $F_{1 p}^{d}$, which is anticipated in several approaches to the description of the dressed quark structure of the nucleon. With the cautionary reminder that these plots are based on an extrapolation of the neutron form factors $F_{1 n}$ and $F_{2 n}$ beyond the range of the data available today, such a zero crossing can be the result of the correlation between an axial- and scalar diquark in the d-quark form factor for the proton, $F_{1}^{d}[29,30]$.

\section{Conclusion}

Recent elastic form factors data have triggered a re-examination of the various QCD based models of the nucleon. New ideas on the way to obtain charge and magnetization distributions have emerged. The possibility that the nucleon is deformed in its ground state, and that it can be described with QCD in the form of solutions of the DysonSchwinger equation, with interesting predictions for the higher $\mathrm{Q}^{2}$ behavior of $G_{E n}$ in particular, are interesting developments.

\section{Acknowledgments}

The author acknowledges grant support from the U.S. National Science Foundation (NSF award no. 1066374).

\section{References}

[1] R. Hofstadter, Rev. Mod. Phys. 28, 214 (1956).

[2] L.L. Foldy, Phys. Rev. 87, 1952.

[3] A. I. Akhiezer and M. P. Rekalo, Sov. J. Part. Nucl. 4, 277 (1974); Fiz. Elem. Chast. Atom. Yadra 4, 662 (1973).

[4] R.G. Arnold, C.E. Carlson, F. Gross, Phys. Rev. C 23, 363 (1981).

[5] C.F. Perdrisat, V. Punjabi and M. Vanderhaeghen, Progress in Particle and Nuclear Physics, 59,694-764 (2007).

[6] C.F. Perdrisat and V. Punjabi, http://www.scholarpedia.org/article/Nucleon Form Factors.

[7] M.K. Jones et al., Phys. Rev. Lett. 84, 1398 (2000). 
[8] O. Gayou et al., Phys. Rev. Lett. 88, 092301 (2002).

[9] V. Punjabi et al. Phys. Rev. C 71 (2005) 055202.

[10] A.J.R Puckett et al, Phys. Rev. Lett. 104, 242301 (2010).

[11] M. Meziane et al. [GEp2gamma Collaboration], Phys. Rev. Lett. 106, 132501 (2011).

[12] A.J.R Puckett et al, Phys. Rev. 85, 045203 (2012).

[13] L. Andivahis et al., Phys. Rev. D 505491 (1994).

[14] M.E.Christy et al., Phys. Rev. C70 015206 (2004).

[15] I.A. Qatan et al., Phys. Rev. Lett. 94142301 (2005).

[16] C.B. Crawford et al. Phys. Rev. Lett.98, 052301 (2007).

[17] G. Ron et al, Phys. Rev. C055204 (2011).

[18] X. Zhan et al, Phys. Lett. B 705,59 (2011).

[19] M. Paolone et al, Phys. Rev. Lett. 105, 072001 (2010)

[20] R. Pohl et al Nature 466, 213 (2010)

[21] P. A. M. Guichon and M. Vanderhaeghen, Phys. Rev. Lett. 91, 142303 (2003).

[22] P.G. Blunden, W.Melnitchouk and J.A.Tjon, Phys. Rev. Lett. 91 (2003) 142304.

[23] Y.-C.Chen, A.Afanasev, S.J.Brodsky, C.E.Carlson, and M.Vanderhaeghen, Phys. Rev. Lett. 93, 122301 (2004); A.V. Afanasev, S.J. Brodsky, C.E. Carlson, Y.C. Chen, M. Vanderhaeghen, Phys. Rev. D 72, 013008 (2005).

[24] N. Kivel and M. Vanderhaeghen, Phys. Rev. Lett. 103, 092004 (2009).

[25] Yu M. Bystritskiy, E.A. Kuraev and E. Tomasi-Gustafsson, Phys. Rev. C 75015207 (2007).

[26] N. Kivel, private communication (2012).

[27] G.D. Cates, C.W. de Jager, S. Riordan and B. Wojtsekhowski, Phys. Rev. Lett. 106 $252003(2011)$

[28] J.J. Kelly, Phys. Rev. C 70, 068202 (2004).

[29] I.C. Cloët and C.D. Roberts, Proc. of Sc. LC2008:047 (2008); arXiv:0811.2018 [nuclth] (2008); I.C. Cloët, G. Eichmann, B. El-Bennich, T. Klähn and C.D. Roberts, Few Body Syst. 46, 1 (2009)

[30] D.J. Wilson, I.C. Cloet, L. Chang and C.D. Roberts, Phys.Rev. C85 (2012) 025205. 